\title{
Review of: "Fe(II)-phthalocyanine supported on chitosan aerogel as a catalyst for oxidation of alcohols and alkyl arenes"
}

\author{
Paulo Menezes ${ }^{1}$ \\ 1 Universidade Federal de Pernambuco
}

Potential competing interests: The author(s) declared that no potential competing interests exist.

This manuscript describes the application of an heterogeneous catalyst based on Chitosan aerogel modified with $\mathrm{Fe}$ (II)-phthalocyanine for the oxidation of alcohols, and alkyl arenes to the corresponding aldehydes and ketones using $\mathrm{H} 2 \mathrm{O} 2$ as an oxidant. The reaction has a high turnover number and the catalyst was well characterized by IR, EDX, SEM micrographs, TGA, etc. The manuscript has some positive points and would be of interest to those in the area, however, the authors should revise the present manuscript considering the following comments:

\section{Introduction:}

All references in this section should be updated. For example, "Nowadays, these materials and reductions, and decomposition of peroxides1". However, the reference cited is dated from 2013 (almost 10 years ago).

\section{Experimental}

2.1 “To a round-bottomed flask containing benzyl alcohol (10 mmol) and FePc@ChA (0.2 g), $11 \mathrm{mmol}$ of $\mathrm{H} 2 \mathrm{O} 2 "$ " The amount in grams of all chemicals as well as the concentration of $\mathrm{H} 2 \mathrm{O} 2$ should be added to the procedure (30\%?).

2.2 "Typical procedure for the oxidation of benzyl alcohol with FePc@ChA". The authors should add some information about a large-scale reaction (For example, a $100 \mathrm{mmol}$ scale). In addition, a reaction example describing the chemical yield (and not only the conversion) should be added. This data would give to the reader some information about the possibility of adsorption of some product on the surface of the heterogeneous catalyst.

2.3 Were the GC analyzes of the products obtained performed with crude products of the reaction? Are they sufficiently pure? Or a purification step was required? For example, the conversion shown in Table 1 (entry 1 ) was $40 \%$. What happened to the rest of the starting material? Was it recovered at the end of the reaction?

\section{Results}


3.1 Table 1. Some additional examples should be added to the table. For example, toluene (PhMe) was used as the reaction substrate?

3.2 For benzylic alcohols, the presence of electron-withdrawing groups gave the corresponding aldehydes in low conversions (Table 1, entries 2 and 3), however, the reaction was not performed using a benzylic alcohol containing an electron-donating group (which could stalilize the radical formed in the proposed mechanism). This would give the reader some additional information.

3.3 The chemoselectivity of the reaction should also be studied. The methodology should be tested using, for example, an aromatic compound containing both an alkyl and alcohol groups (or a competitive experiment using both substrates from entries 1 and 7 of Table 1). Moreover, does the reaction tolerates protecting groups (such as TBS or THP)? This is a crucial information for Organic Chemists.

3.4 As the authors proposed a "radical mechanism" a comparative experiment using a radical scavenger such as TEMPO should be performed and the results inserted in the main text of the manuscript.

Apparently, the manuscript contains important information. However, a MAJOR REVISION of it is necessary to meet the criteria of Scientific Reports. 